\title{
SOIL MOISTURE ACTIVE/PASSIVE (SMAP) RADIOMETER SUBBAND CALIBRATION AND CALIBRATION DRIFT
}

\author{
Jinzheng Peng ${ }^{1,2}$, Jeffrey R. Piepmeier ${ }^{1}$, Giovanni De Amici ${ }^{1}$, Priscilla N. Mohammed ${ }^{1,3}$, \\ ${ }^{1}$ NASA's Goddard Space Flight Center, Greenbelt, MD USA \\ ${ }^{2}$ Universities Space Research Association, MD USA \\ ${ }^{3}$ Morgan State University, Baltimore, MD USA
}

\section{INTRODUCTION}

The SMAP is one of four first-tier missions recommended by the US National Research Council's Committee on Earth Science and Applications from Space (Earth Science and Applications from Space: National Imperatives for the Next Decade and Beyond, Space Studies Board, National Academies Press, 2007) [1]. The observatory was launched on Jan 31, 2015. The goal of the SMAP is to measure the global soil moisture and freeze/thaw from space. The L-band radiometer is the passive portion of the spaceborne instrument. It measures all four Stokes antenna temperatures and outputs counts. The Level 1B Brightness Temperature (L1B_TB) science algorithm converts radiometer counts to the Earth's surface brightness temperature [2]. The results are reported in the radiometer level $1 \mathrm{~B}$ data product together with the calibrated antenna temperature (TA) and all of the corrections to the unwanted sources' contribution. The calibrated L1B data product are required to satisfy the overall radiometer error budget of $1.3 \mathrm{~K}$ needed to meet the soil moisture requirement of 0.04 volumetric fraction uncertainty and the calibration drift requirement of no larger than $0.4 \mathrm{~K}$ per month.

Two

\section{SUBBAND CALIBRATION}

The SMAP radiometer is a fully polarimetric radiometer which measures all of the 4 Stokes parameters. For Radio Frequency Interference (RFI) detection and mitigation purpose, the radiometer digital electronics (RDE) samples receiving signals and computes the full Stokes vectors within the 24-MHz full-band channel and $161.5-\mathrm{MHz}$ subband channels besides the first 4 signal moments for further ground processing. Due to downlink bandwidth limit, the subband data only be downloaded when the spacecraft is over land, Dome-C, and a small ocean region within the Pacific ocean. Ground processing algorithm reports calibration antenna temperature into the radiometer L1B data product using subband Stokes vectors if they are available, otherwise, full band Stokes vectors will be used. When subband Stokes vector is available, the reported TA is the mean value of the RFI-free subband TAs. (Note: the adjacent subbands to the RFI-detected subband are treated as RFI contaminated.) Therefore, the subband TAs needs to be as close to the full band TA as possible.

Two subband calibration approaches have been used in the SMAP radiometer subband calibration. The first one is that all of the TAs in the subbands and fullband are compared to the expected TA over global 
ocean shown in Figure 1. The second approach uses the full band TA to calibrate all of the subbands. Each approach has its own advantage/disadvantage. Their performance will be presented.

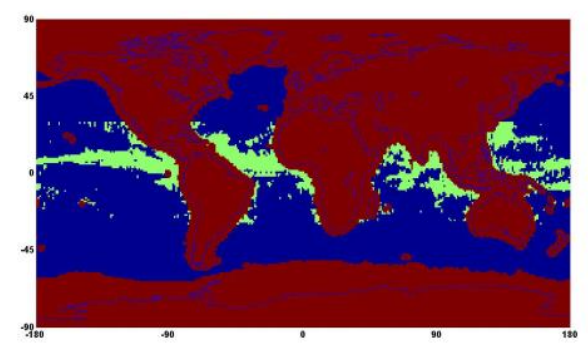

Figure 1 Global ocean mask (in blue) for calibration and drift monitoring

\section{EXPECTED ANTENNA} TEMPERATURE MODEL

We have developed the SMAP level 1B forward brightness temperature simulator which computes the expected antenna temperature using two-dimensional integration over all $4 \pi$ steradians of antenna pattern but the real-time computation cannot be achieved. A simplified version is then developed without the two-dimensional integration, but the unwanted sources' impacts are still considered with following features:

1) The Earth's land surface TBs are from Nature Run v03 data product [3]. For ocean and ice, L-band GMF model [4] and Ulaby et al model [5] are used, respectively.

2) The simulated contributions from the Sun/Moon/Galaxy are the same as that used in the SMAP radiometer L1B algorithm except that the Earth's surface reflectivity is from the Earth surface model.
3) The antenna temperature from the Earth's sidelobe is estimated by using the inverted matrix of the APC transformation matrix used in the SMAP radiometer L1B algorithm.

4) The atmospheric radiative transfer model is Earth surface elevation dependent. Although its accuracy is not as good as the model [6] used in the radiometer L1B algorithm, it still satisfies its $0.1 \mathrm{~K}$ error budget allocation. The upwelling brightness temperature and loss factor are modeled as

$$
\hat{T}_{u p}=0.0400 H^{2}-0.5442 H+2.7755
$$

$$
\hat{L}=1.6495 \times 10^{-4} H^{2}-0.0021 H+1.0109
$$

where $\mathrm{H}$ is the elevation of the Earth's surface, in $\mathrm{km}$.

5) The total electron content (TEC) for Faraday rotation angle computation is obtained from the International GNSS Service (IGS) [7].

\section{CALIBRATION DRIFT}

The primary method of monitoring the calibration drift is to compare the measured antenna temperature to the expected antenna temperature on a globally and daily averaged basis. With this method, it was observed that the measured antenna temperatures were drifting compared to the expected antenna temperatures over global ocean during the first two month, eclipse season, and the period of SAR transmitter disable. The solid lines in Figure 2 show the drift in the measured antenna temperature 
using constant calibration coefficients. During April 03 13, 2015, the SMAP radar transmitter was disabled, and it caused the measured antenna temperatures decreased by $1 \mathrm{~K}$ for $\mathrm{V}$-pol and $1.4 \mathrm{~K}$ for $\mathrm{H}$-pol. The mechanism of the TA decrement is still under investigation.

After the SMAP radar transmitter enable and before May 27, 2015, the measured TAs were drifting at approximately constant rate. It's most likely due to outgassing in vacuum environment and any thermal drifts with the spacecraft entering eclipse.

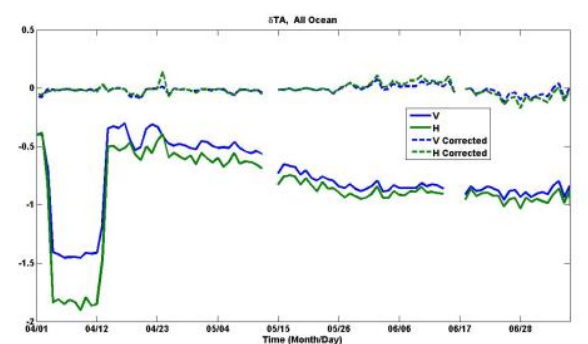

Figure 2. Calibration drift

The drift with the bias in Figure 2 can be removed by adjusting the brightness temperature of the radiometer internal noise source daily. This method has been applied in the SMAP radiometer L1B data product released in September, 2015. The performance is shown in dash lines in Figure 2. Calibration drift is around zero after Tnd adjusted (daily if necessary) . The RMSE in the Earth's ocean surface brightness temperature is $1.3 \mathrm{~K}$ for $\mathrm{V}$-pol and $1.27 \mathrm{~K}$ for H-pol evaluated by the global ocean target.

\section{CONCLUSION}

The radiometer Subband calibration and calibration drift correction have been successfully used in the released radiometer L1B data product. Although their performances satisfy the requirements, they are still under continuing analysis to find their remaining uncertainty. The progress will be presented besides the current performance. 